\title{
Systematic and Endothelial Inflammation and Endothelial Progenitor Cell Levels in Emphysematous Rats Exposed to Intermittent Hypoxia
}

\author{
Qing-chan Yang MD, Xin Sun MD, Yong-mei Wang MD, Qi Wu MD, \\ Jing Feng MD PhD, and Bao-yuan Chen MD
}

\begin{abstract}
BACKGROUND: This study aimed to develop an overlap syndrome rat model with intermittent hypoxia (IH) exposure as seen in obstructive sleep apnea, on a base of preexisting emphysema caused by $16 \mathrm{wk}$ of smoke exposure to determine whether IH and emphysema existing simultaneously play overlapped roles on systematic/endothelial inflammation and endothelial damage. METHODS: Sixty male Wistar rats were divided into 4 groups of $\mathbf{1 5}$ each, labeled according to exposure conditions as control, IH, emphysema, and overlap groups. In these animals, electroencephalogram monitoring and preliminary experiments to obtain arterial blood gas values were performed. Serum concentrations of tumor necrosis factor (TNF)- $\alpha$ and interleukin (IL)-6, TNF- $\alpha$ and IL-6 concentrations in the culture medium, Ras homology A mRNA expression levels of endothelial cells from right common carotid artery, and ratio of carotid intima-media thickness of whole thickness of vascular wall expressed in percent (C-IMT) $(\%)$ values were evaluated. Subsequently, circulating endothelial progenitor cells (EPCs) within rat peripheral blood and bone marrow were measured with flow cytometry. RESULTS: The serum and endothelial concentrations of TNF- $\alpha$ and IL-6 and the levels of endothelial Ras homology A mRNA have statistically significant results described as overlap > emphysema > IH > control. The levels of EPCs in rat peripheral blood and bone marrow have statistically significant results described as overlap $>$ IH $>$ emphysema > control. C-IMT (\%) values from right common carotid artery are the highest in the overlap group and the lowest in the control group. There is no statistical difference when comparing the IH and the emphysema groups. CONCLUSIONS: Regardless of whether IH and emphysema exposure are mechanistically synergistic, this overlap elicits a more severe systematic/endothelial inflammation and endothelial damage; meanwhile, a robust mobilization of EPCs is demonstrated, which is not to mean a robust adherent and repairing capability. Key words: intermittent hypoxia; emphysema; inflammation; endothelial dysfunction; endothelial progenitor cell. [Respir Care 2015;60(2):279-289. @ 2015 Daedalus Enterprises]
\end{abstract}

\section{Introduction}

Obstructive sleep apnea (OSA) and COPD are 2 of the most prevalent chronic respiratory disorders. COPD occurs in $\sim 10-20 \%$ of patients with OSA, ${ }^{1}$ whereas there is

\footnotetext{
Drs Yang, Sun, Wang, and Wu are affiliated with the Tianjin Haihe Hospital, Tianjin, China. Drs Feng and Chen are affiliated with the Respiratory Department, Tianjin Medical University General Hospital, Tianjin, China. Dr Feng is also affiliated with the Neuropharmacology Section, Laboratory of Toxicology and Pharmacology, National Institute of Environmental Health Sciences, National Institutes of Health, Research Triangle Park, North Carolina.
}

Drs Yang and Sun are co-first authors of this paper. considerable controversy regarding the prevalence of OSA in patients with COPD, with reported values ranging up to approximately $15 \%$ of COPD patients. ${ }^{2}$ The coexistence of OSA and COPD is referred to as the respiratory overlap

\footnotetext{
Supplementary material related to this paper is available at http:// www.rcjournal.com.

This study was supported by grants 81270144,30800507 , and 81170071 from the National Natural Science Foundation of China; by grants $13 J C Y B J C 22400$ and 13JCYBJC40000 from the Natural Science Foundation of Tianjin City; and by grant 09ZCZDSF04500 from the Tianjin Municipal Science and Technology Commission. The authors have disclosed no conflicts of interest.
} 
syndrome (OS). ${ }^{3}$ Epidemiologic studies show a prevalence of OS of approximately $1 \%$ in adult males, but the coexistence of asymptomatic COPD and OSA is probably much higher. ${ }^{4}$ COPD includes 2 major subtypes, chronic bronchitis and emphysema. The emphysema subtype, composing most of the pathophysiologic components of COPD, seems to present more obvious systemic inflammation, suggesting that it may be more prone to systemic consequences of COPD compared with subtypes without emphysema. Because of simultaneous occurrence, it is very difficult to differentiate the effects of its 2 main pathologic traits, which are intermittent hypoxia (IH) and sleep fragmentation, in clinical and bench studies of OSA. Many OSA studies utilize IH, a major initiator of OSA inflammatory pathways, as the only exposure factor. These approaches simplify the research process and attain most of the academic goals. . $^{5-9}$

Cardiovascular diseases represent a major comorbidity in each disorder of OS. Both COPD and OSA are associated with increased activation of many inflammatory cells and molecular mechanisms associated with atherosclerosis, which provide basic mechanisms to support the clinical and epidemiological data demonstrating COPD and OSA as independent risk factors for cardiovascular diseases. ${ }^{10}$ Endothelial dysfunction from systemic and endothelial inflammation develops at early stages involving tumor necrosis factor- $\alpha$ (TNF- $\alpha$ ) and interleukin-6 (IL-6). This process promotes rolling and adherence of leukocytes to the endothelium. Assisted by endothelial cell Ras homology A (RhoA), ${ }^{11}$ monocytes and $\mathrm{T}$ cells penetrate the vascular wall, where monocytes transform into macrophages and ingest oxidized lipids to form foam cells, which is a key process of early atherosclerosis and subsequent cardiovascular diseases. ${ }^{10}$ Circulating endothelial progenitor cells (EPCs), which are thought to originate from myeloid pluripotent stem cells, after being mobilized from bone marrow into circulating blood, provide an endogenous repair mechanism to counteract ongoing endothelial cell injury, replace dysfunctional endothelium, and enhance tissue repair after ischemic vascular injury. ${ }^{12}$

OSA and COPD are associated with systemic and endothelial inflammation, and both are independent risk factors for the development of cardiovascular disease. ${ }^{13}$ Thus, the question of whether having OSA or COPD simultaneously plays overlapped roles on systematic/endothelial inflammation and endothelial dysfunction is an interesting

Correspondence: Jing Feng MD PhD, Respiratory Department, Tianjin Medical University General Hospital, Tianjin 300052, China. E-mail: zyyhxkfj@126.com. Yong-mei Wang MD, Tianjin Haihe Hospital, Tianjin 300350, China. E-mail: tjshhyywym@126.com.

DOI: $10.4187 /$ respcare. 03492

\section{QUICK LOOK}

\section{Current knowledge}

COPD and obstructive sleep apnea co-exist in 15-20\% of patients. The impact of intermittent hypoxia (IH) associated with sleep apnea on systemic inflammation and progression of disease is difficult to study in humans and is not well described.

\section{What this paper contributes to our knowledge}

In a rat model, regardless of whether the IH and emphysema exposure are mechanistically synergistic, this overlap elicits a more severe systematic/endothelial inflammation and endothelial damage. Mobilization of circulating endothelial progenitor cells was seen, which may explain pulmonary vascular changes in this population.

one. ${ }^{14}$ To shine some insight on that point, in this study, an OS rat model was developed with IH exposure in addition to preexisting emphysema caused by $16 \mathrm{wk}$ of smoke exposure. In different rat groups, systemic inflammation, endothelial inflammation, endothelial RhoA mRNA, and the ratio of carotid intima-media thickness to whole thickness of the vascular wall (C-IMT) were evaluated. Next, EPCs within rat bone marrow and peripheral blood, a reservoir of cells that could be home to sites of injury and restore endothelial integrity or normal function, ${ }^{15}$ were measured to assess the repairing competence after potential inflammatory damage.

\section{Methods}

\section{Overall Rat Model Experimental Protocol}

The overall rat model experimental protocol (overlap exposure) was IH exposure in addition to preexisting emphysema, which was caused by 16 wk of smoke exposure followed by the bench studies on these animals. To minimize the suffering of animals during the experiment and maximize the information from our animal study, we designed, analyzed, and reported this research referring to the ARRIVE Guidelines. ${ }^{16,17}$ Nine 9 AM to 5 PM is the sleeping time for Wistar rats, which were used in our study. According to our previous studies, ${ }^{7,8}$ hypoxia treatment from 9 AM to 5 PM in mild to moderate severity will not stress Wistar rats, and cigarette smoke exposure is not uncomfortable for rats. The institutional review board of Tianjin Medical University General Hospital approved the ethical and methodological aspects of the investigation (approval EA-20120002.). Sixty male Wistar rats weighing $\sim 120-150 \mathrm{~g}$ at $4 \mathrm{wk}$ of age (provided by the Model 
Animal Center, Radiological Medicine Research Institute, Chinese Academy of Medical Science, Tianjin, People's Republic of China; license SCXK Tianjin 2010-0002) were divided into 4 groups of 15 each according to exposure conditions as follows: (1) control group $(n=15)$, sham smoke exposure and sham IH exposure; (2) IH group $(n=15)$, sham smoke exposure and true IH exposure; (3) emphysema group $(n=15)$, true smoke exposure and sham IH exposure; and (4) overlap group $(n=15)$, true smoke exposure and true IH exposure.

\section{Animal Cigarette Smoke Exposure}

Animals were housed in standard laboratory cages (5 per cage) and allowed food and water ad libitum. We used a cigarette smoke exposure device similar to those used in our previous studies..$^{7,8}$

\section{Animal IH Exposure}

Model rats were exposed to $\mathrm{IH}$ for $4 \mathrm{wk}$, from 9 AM to 5 PM daily, from the start of wk 13 of cigarette smoke exposure; meanwhile, cigarette smoke exposure was continued for model rats. This means that rats were exposed to smoke exposure and $\mathrm{IH}$ simultaneously from the start of wk 13 to the end of wk 16 (4 wk). We used an IH exposure device similar to those used in our previous studies. ${ }^{6,8}$ Briefly, a gas control delivery system was designed to regulate the flow $(5 \mathrm{~L} / \mathrm{min})$ of nitrogen $\left(\mathrm{N}_{2}, \mathrm{IH}\right.$ phase) or clean air (air, normoxia phase) alternatively into a customized IH housing chamber $(23 \mathrm{~cm} \times 20 \mathrm{~cm} \times 12 \mathrm{~cm}=$ $5,520 \mathrm{~cm}^{3} \approx 5.5 \mathrm{~L}$ ) to maintain a designated IH or normoxia environment. A series of programmable solenoids and flow regulators altered the fractional concentration of inspired oxygen that controlled the durations of hypoxia (30 s) or normoxia phase (90 s) and produced 30 cycles of alternations/h, and the nadir oxygen concentration in the IH housing chamber was approximately $5 \% . \mathrm{O}_{2}$ and $\mathrm{CO}_{2}$ concentration analyzers (Hamilton, Bonaduz, Switzerland) were used to monitor the environment in the housing chamber for rats. The sham IH exposure rats were exposed to a sham environment; otherwise, conditions were the same except that the nitrogen source was changed to a clean air source.

\section{Animal Electroencephalogram Monitoring and Preliminary Experiments to Obtain Arterial Blood Gas Values}

One week before the experimental exposure, 5 rats were selected randomly from each group. Two stainless steel screws attached to insulated wires were implanted in the skull of each rat (from bregma: anteroposterior, $+2 \mathrm{~mm}$, mediolateral, $-2 \mathrm{~mm}$; and anteroposterior, $-3 \mathrm{~mm}$, me- diolateral, $+2 \mathrm{~mm}$ ) to record electroencephalogram. ${ }^{18}$ For electrophysiological recording, a lightweight shielded cable was connected to the plug on the rats' heads, allowing free movement of the rats within the cage. The signals were routed to an electroencephalograph (Nicolet Bravo, Natus Medical, San Carlos, California) so that sleep stages could be monitored conveniently and continuously.

Two days before the end of exposure period (end of wk $16-2 \mathrm{~d}$ ), these rats with wires were cannulated to obtain arterial blood gas (ABG) data with the method used in our previous studies. ${ }^{7}$ Briefly, after light anesthetization (10\% chloral hydrate, $0.1 \mathrm{~mL} / 100 \mathrm{~g}$ body weight intraperitoneally), the right femoral artery of selected rats was cannulated to obtain blood samples for ABG testing at any time as necessary. At the end of treatment (end of wk 16), arterial blood samples $(0.7 \mathrm{~mL}$ each) were drawn under the following conditions:

1. Slow wave sleep period in the control group with clean air exposure

2. Slow wave sleep period in the emphysema group with clean air exposure

3. Nadir partial oxygen pressure $\left(\mathrm{P}_{\mathrm{aO}_{2}}\right)$ point during hypoxia phase of slow wave sleep period in the IH group with IH exposure

4. Zenith $\mathrm{P}_{\mathrm{aO}_{2}}$ point during normoxia phase of slow wave sleep period in the IH group with IH exposure

5. Nadir $\mathrm{P}_{\mathrm{aO}}$ point during hypoxia phase of slow wave sleep period in the overlap group with IH exposure

6. Zenith $\mathrm{P}_{\mathrm{aO}}$ point during normoxia phase of slow wave sleep period in the overlap group with $\mathrm{IH}$ exposure

For each blood sample collected, ABG analysis included measurements of $\mathrm{P}_{\mathrm{aO}_{2}}$, partial carbon dioxide pressure $\left(\mathrm{P}_{\mathrm{aCO}_{2}}\right)$, arterial oxygen saturation, and $\mathrm{pH}$ value.

\section{Anesthetization and Blood Collection}

At the end of exposure, for the other 10 rats of each group, animals were anesthetized intraperitoneally with $10 \%$ chloral hydrate at a dose of $0.3 \mathrm{~mL} / 100 \mathrm{~g}$ of body weight. Rats were killed by carbon dioxide inhalation and exsanguination. During the operation, the body was fixed on the operation table in supine position, and temperature was maintained at $37^{\circ} \mathrm{C}$ with a heat lamp or a heating pad as required. Blood (approximately $8-10 \mathrm{~mL} / \mathrm{rat}$ ) was collected (exsanguination) from the caudal vena cava into a 10 -mL syringe containing $200 \mu \mathrm{L}$ of $10 \%$ weight/volume disodium ethylenediaminetetraacetic acid (VWR Scientific Products, West Chester, Pennsylvania). Syringes were rocked at room temperature until processed $(<1 \mathrm{~h}){ }^{7}$

\section{TNF- $\alpha$ and IL-6 Concentrations in Rat Peripheral Blood}

TNF- $\alpha$ and IL- 6 protein levels in $2 \mathrm{~mL}$ of ethylenediaminetetraacetic acid-treated rat peripheral blood were mea- 
sured with an enzyme-linked immunosorbent assay kit (Jingmei, Shenzhen, People's Republic of China). The remaining rat peripheral blood was kept for the isolation of peripheral blood mononuclear cells.

\section{TNF- $\alpha$ and IL-6 Concentrations in the Culture Medium and RhoA mRNA Expression Levels of Endothelial Cells From Right Common Carotid Artery}

The right common carotid artery of each rat was harvested after systematic exposure, and endothelial cell layers were obtained with a cell scraper from longitudinallycut outspread vessels. Endothelial cells scraped from each preparation were washed twice with ice-cold phosphatebuffered saline, the cells were resuspended with RPMI1640 medium, and cell concentration was adjusted to $3 \times$ $10 \% \mathrm{~mL}$. Endothelial cells were then transplanted to Petri dishes with $1 \mathrm{~mL} /$ well and cultured for $2 \mathrm{~h}$ in a standard incubator. TNF- $\alpha$ and IL-6 protein levels in the culture medium were measured with an enzyme-linked immunosorbent assay kit (Jingmei). Total RNA was extracted from adherent cells pellet centrifuged after resuspending, and phosphatebuffered saline washing. Reverse transcription polymerase chain reaction was used to analyze endothelial cell RhoA mRNA expression levels (RhoA: sense primer, 5'-ATGTGCCCACAGTGTTTGAGAAC-3', antisense primer, 5' TCAGTTCGTAAAGACAGGGTTGC-3'; glyceraldehyde3-phosphate dehydrogenase: sense primer, 5'TATTGGGCGCCTGGTCACCA-3', antisense primer, 5' CCACCTTCTTGATGTCATCA-3' [Invitrogen]; RhoA cDNA product, $345 \mathrm{bp}$; glyceraldehyde-3-phosphate dehydrogenase cDNA product, $726 \mathrm{bp}$ ). Optical density values of reverse transcription polymerase chain reaction results were standardized with the results of the control group. ${ }^{6}$

\section{Histological Evaluation of the Lung and Right Common Carotid Artery}

All right lungs were inflated intrabronchially (from the right primary bronchus) with $10 \%$ formalin at a constant transpulmonary pressure of $25 \mathrm{~cm}$ of formalin for at least $18 \mathrm{~h}$ with the right lungs impregnated wholly in the formalin at the same time. Next, a little part of right common carotid artery of each rat was fixed. The fixed tissues were embedded in paraffin wax after tissue processing. Sections (4- $\mu \mathrm{m}$ thickness) were cut from the paraffin block and stained with hematoxylin/eosin. Images of each section were captured at $40 \times$ (lung) and $40 \times$ (right carotid artery), with a Baumer Optronic digital camera through an Olympus BX40 microscope (Olympus Optical, Tokyo, Japan) and were saved as JPEG files with Zeiss KS300 image-processing software (Imaging Associates, Bicester, United Kingdom). ${ }^{8}$ Ratio of carotid intima-media thick- ness of whole thickness of vascular wall expressed in percent (C-IMT) (\%) was evaluated.

\section{Measurement of EPCs From Rat Peripheral Blood and Bone Marrow}

Peripheral blood mononuclear cells were isolated from rat peripheral blood by light density separation using Histopaque 1083 (Sigma-Aldrich, Thermo Fisher Scientific, Waltham, Massachusetts; $1.083 \mathrm{~g} / \mathrm{mL}$ at $25^{\circ} \mathrm{C}$ ) according to the instructions supplied by the manufacturer. Isolation and culture of mononuclear cells from rat bone marrow were also performed. ${ }^{19}$ The EPCs from rat peripheral blood and bone marrow were measured by a FACScalibur flow cytometer (Becton Dickinson, San Jose, California) and analyzed using Cell-Quest and Paint-a-Gate software (Becton Dickinson). EPCs from peripheral blood are expressed as the number of collected peripheral blood mononuclear cells $/ \mu \mathrm{L}$ of whole blood $\times$ EPC percentage $($ cells $/ \mu \mathrm{L})$, namely, the concentrations of EPC in rat peripheral blood, when EPCs of bone marrow are expressed as percentages of mononuclear cells (\%).

\section{Statistical Analysis}

SPSS 18.0 (SPSS, Chicago, Illinois) software package was used for statistical analysis and illustration. One-way analysis of variance was performed for whole difference among groups, and Bonferroni post hoc multiple comparisons were used to evaluate differences between internal groups. Unless otherwise stated, values were reported as mean $\pm \mathrm{SD}$, and $P<.05$ is considered statistically significant.

\section{Results}

Table 1 summarizes the $\mathrm{ABG}$ results obtained from preliminary experiments at the end of exposure period. The values in this table are descriptive, not for data comparison among groups. In the control group, the $\mathrm{P}_{\mathrm{aO}}$ in slow wave sleep period is $94.3 \pm 2.6 \mathrm{~mm} \mathrm{Hg}$ as expected, and the $\mathrm{P}_{\mathrm{aO}_{2}}$ value of the IH group in hypoxia phase or normoxia phase of slow wave sleep period is $51.5 \pm 1.2$ or $90.2 \pm$ $4.0 \mathrm{~mm} \mathrm{Hg}$, respectively. Accordingly, the $\mathrm{P}_{\mathrm{aO}}$ of the emphysema group in slow wave sleep period is $73.0 \pm$ $2.2 \mathrm{~mm} \mathrm{Hg}$, and the $\mathrm{P}_{\mathrm{aO}_{2}}$ value of the overlap group in hypoxia phase or normoxia phase is $45.4 \pm 2.3$ or $73.0 \pm$ $2.1 \mathrm{~mm} \mathrm{Hg}$, respectively.

\section{Histological Evaluation of the Lung}

Figure 1A shows the pathologic images of lung section randomly selected from various groups. Pathologic char- 
Table 1. Descriptive ABG Results From Preliminary Tests

\begin{tabular}{|c|c|c|c|c|c|c|}
\hline & \multirow{2}{*}{ Control } & \multirow{2}{*}{ Emphysema } & \multicolumn{2}{|c|}{$\mathrm{IH}$} & \multicolumn{2}{|c|}{ Overlap } \\
\hline & & & IH-H & IH-R & Overlap-H & Overlap-R \\
\hline $\mathrm{P}_{\mathrm{aO}_{2}}(\mathrm{~mm} \mathrm{Hg})$ & $94.3 \pm 2.6$ & $73.0 \pm 2.2$ & $51.5 \pm 1.2$ & $90.2 \pm 4.0$ & $45.4 \pm 2.3$ & $73.0 \pm 2.1$ \\
\hline $\mathrm{P}_{\mathrm{aCO}_{2}}(\mathrm{~mm} \mathrm{Hg})$ & $41.9 \pm 2.3$ & $48.9 \pm 2.9$ & $37.0 \pm 0.9$ & $40.7 \pm 3.2$ & $42.3 \pm 3.2$ & $48.4 \pm 2.4$ \\
\hline $\mathrm{S}_{\mathrm{aO}_{2}}(\%)$ & $97.1 \pm 0.3$ & $91.8 \pm 0.6$ & $86.9 \pm 1.8$ & $95.3 \pm 1.6$ & $83.7 \pm 2.8$ & $91.8 \pm 0.6$ \\
\hline $\mathrm{pH}^{2}$ & $7.41 \pm 0.01$ & $7.36 \pm 0.01$ & $7.42 \pm 0.01$ & $7.42 \pm 0.01$ & $7.41 \pm 0.01$ & $7.36 \pm 0.01$ \\
\hline $\begin{array}{l}\mathrm{ABG}=\text { arterial blood } \\
\mathrm{IH}=\text { intermittent hyp } \\
\mathrm{H}=\text { hypoxia phase } \\
\mathrm{R}=\text { reoxygenation ph } \\
\mathrm{S}_{\mathrm{aO}_{2}}=\text { arterial oxyhe }\end{array}$ & uration & & & & & \\
\hline
\end{tabular}
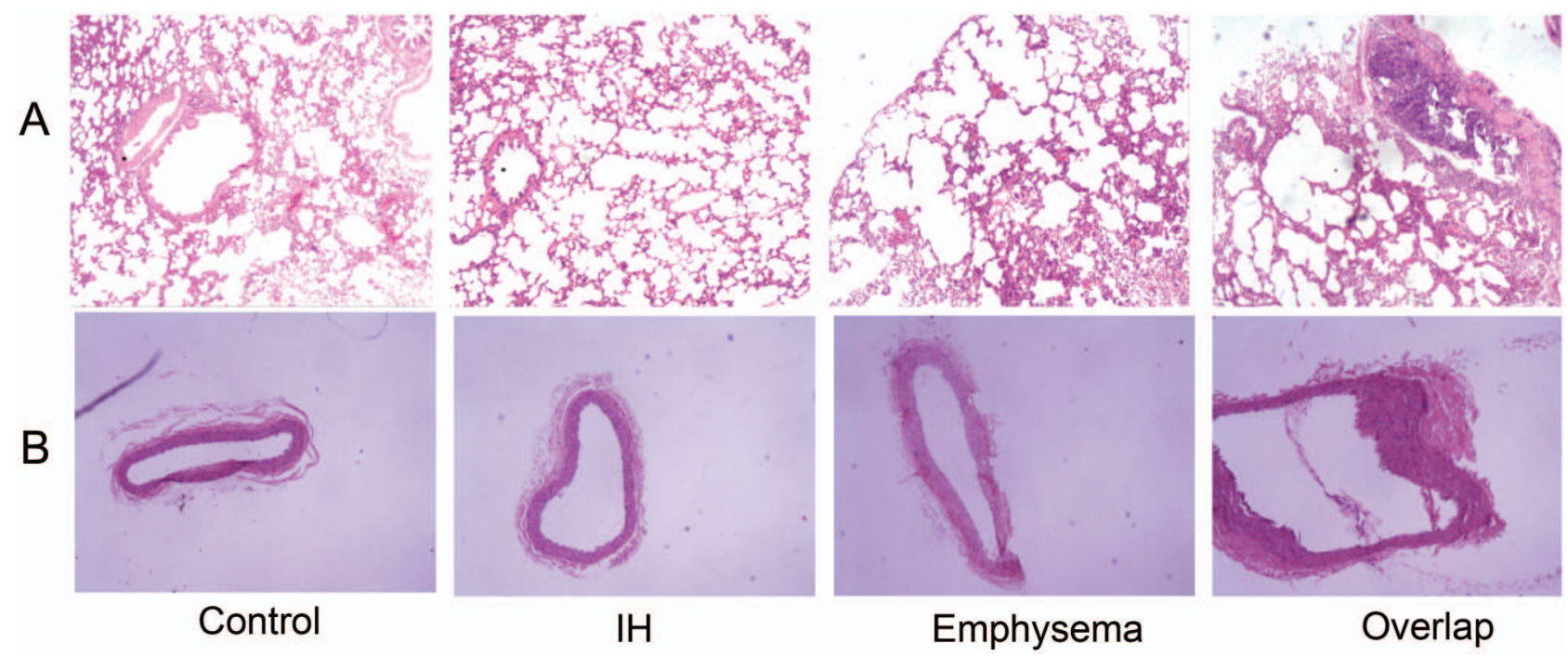

Fig. 1. Histological photographs from different groups. A and B are histological photographs from lung and right carotid artery, stained with hematoxylin and eosin and captured with $40 \times$ light microscopy. $\mathrm{IH}=$ intermittent hypoxia.

acteristics of emphysema were obvious in those rats from the emphysema and overlap groups, including inflammatory cell infiltration, increased mean linear intercept, and decreased mean alveolar number per microscope field. For details, see the supplementary materials at http://www. rcjournal.com.

\section{Serum Concentrations of TNF- $\alpha$ and IL-6}

TNF- $\alpha$ and IL-6 serum concentrations in ethylenediaminetetraacetic acid-treated rat peripheral blood are highest in the overlap group $(76.9 \pm 7.8$ and $683.5 \pm 20.2$ $\mathrm{pg} / \mathrm{mL}$, respectively, all $P$ values $<.001)$ and lowest in the control group $(16.5 \pm 2.3$ and $109.7 \pm 7.8 \mathrm{pg} / \mathrm{mL}$, respectively, all $P$ values $<.001)$. When comparing the emphysema and IH groups, serum TNF- $\alpha$ and IL-6 levels in the emphysema group are higher $(62.6 \pm 4.8$ vs $48.6 \pm 3.2$ $\mathrm{pg} / \mathrm{mL}$ for TNF- $\alpha$ and $478.6 \pm 12.2$ vs $264.6 \pm 16.7$ $\mathrm{pg} / \mathrm{mL}$ for IL-6, respectively, all $P$ values $<.001)$. See Figure 2.

\section{TNF- $\alpha$ and IL-6 Relative Concentrations in the Culture Medium of Endothelial Cells From Right Common Carotid Artery}

TNF- $\alpha$ and IL- 6 relative concentrations in the culture medium of endothelial cells from the right common carotid artery are highest in the overlap group $(188.5 \pm 11.4$ and $1268.7 \pm 35.6 \mathrm{pg} / \mathrm{mL} / 100 \mathrm{mg}$ of protein, respectively, all $P$ values $<.001$ ) and lowest in the control group $(37.6 \pm 4.0$ and $434.6 \pm 16.1 \mathrm{pg} / \mathrm{mL} / 100 \mathrm{mg}$ of protein, respectively, all $P$ values $<.001$ ). When comparing the emphysema and IH group, medium TNF- $\alpha$ and IL-6 levels in the emphysema group are higher $(143.6 \pm 5.6$ vs $112.9 \pm 13.9 \mathrm{pg} / \mathrm{mL} / 100 \mathrm{mg}$ of protein for TNF- $\alpha$ and $779.5 \pm 21.0$ vs $675.3 \pm 15.0 \mathrm{pg} / \mathrm{mL} / 100 \mathrm{mg}$ of protein, respectively, all $P$ values $<.001)$. See Figure 3 .

\section{RhoA mRNA Expression Levels of Endothelial Cells From Right Common Carotid Artery}

RhoA mRNA expression levels shown as standardized optical density values in endothelial cells from right com- 

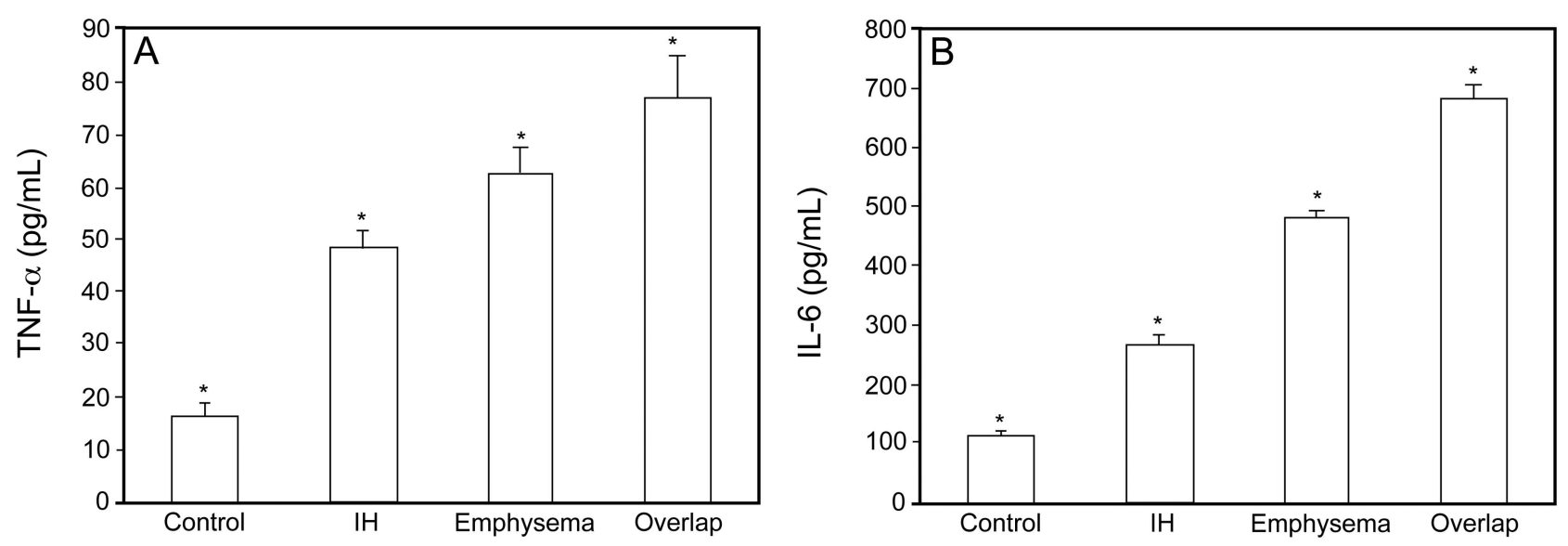

Fig. 2. Serum concentrations of TNF- $\alpha$ and IL-6 from different groups. ${ }^{*} P<.05$ compared with other group. TNF- $\alpha=$ tumor necrosis factor $\alpha$; IL-6 = interleukin-6; IH = intermittent hypoxia.
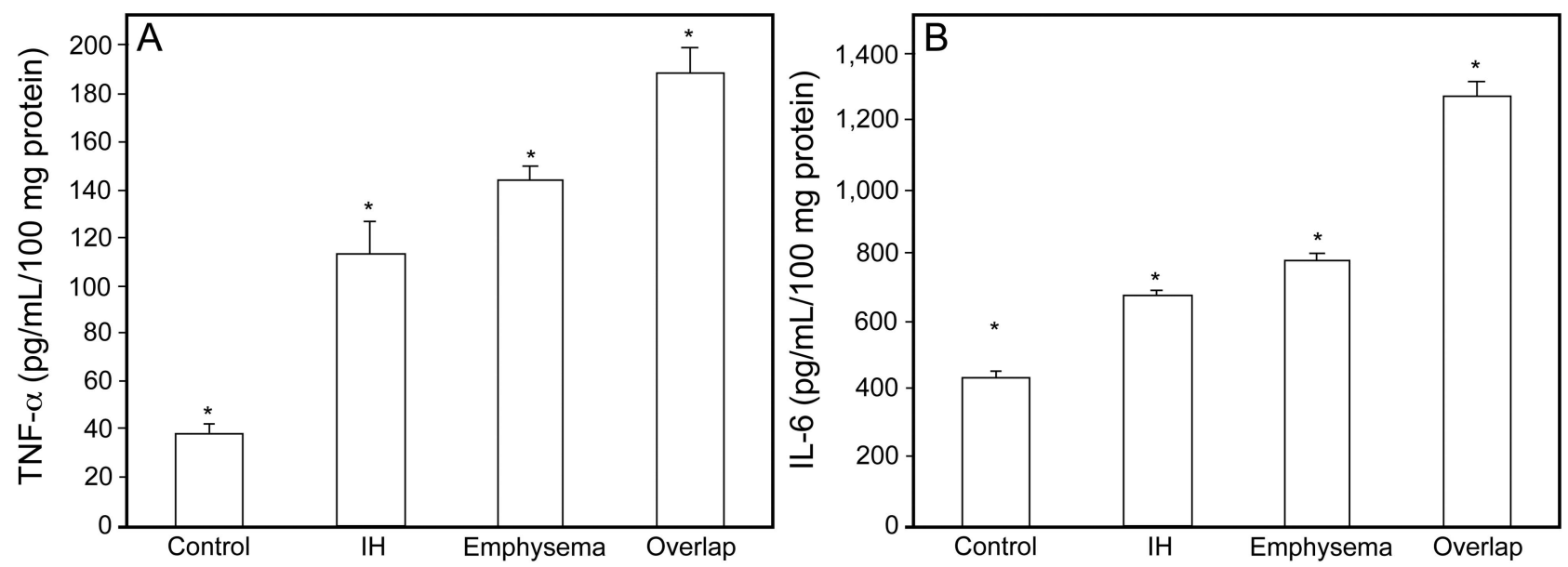

Fig. 3. TNF- $\alpha$ and IL- 6 relative concentrations in the culture medium of endothelial cells from the right common carotid artery of different groups. ${ }^{*} P<.05$ compared with other group. TNF- $\alpha=$ tumor necrosis factor $\alpha$; IL-6 = interleukin-6; IH = intermittent hypoxia.

mon carotid artery are the highest in the overlap group $(2.8 \pm 0.1$, all $P$ values $<.001)$ and the lowest in the control group $(1.0 \pm 0.1$, all $P$ values $<.001)$. When comparing the emphysema and IH group, RhoA mRNA expression levels in the emphysema group $(2.1 \pm 0.2)$ are higher $(1.6 \pm 0.1, P<.001)$. See Figure 4 .

\section{C-IMT (\%) of Right Carotid Artery}

C-IMT (\%) values from right common carotid artery are the highest in the overlap group $(62.5 \pm 3.6 \%$, all $P$ values $<.001)$ and the lowest in the control group $(48.8 \pm 1.3 \%, P$ values $=.003,<.001$, and $<.001$ when compared with $\mathrm{IH}$, the emphysema, and overlap groups, respectively.). There is no statistical difference when comparing IH $(53.4 \pm 2.0 \%)$ and the emphysema group $(56.6 \pm 3.1 \%, P=.061)$. See Figure 5 .

\section{The Concentrations of EPC in Rat Peripheral Blood}

The concentrations of EPC in peripheral blood are the most in the overlap group $(8.89 \pm 0.59$ cells $/ \mu \mathrm{L}$, all $P$ values $<.001)$ and the least in the control group $(4.50 \pm 0.43$ cells $/ \mu \mathrm{L}$, all $P$ values $<.001)$. When comparing the $\mathrm{IH}$ and emphysema groups, concentrations of EPC are higher in the IH group $(7.21 \pm 0.59)$ than the emphysema group (5.85 $\pm 0.43, P<.001)$. See Figure 6 , A and C.

\section{EPC Percentages of Mononuclear Cells in Rat Bone Marrow}

EPC percentages of mononuclear cells in rat bone marrow are the highest in the overlap group $(0.63 \pm 0.05, P$ values $<.001,=.05$, and $<.001$ when compared with the control, IH, and emphysema groups, respectively.) and the 

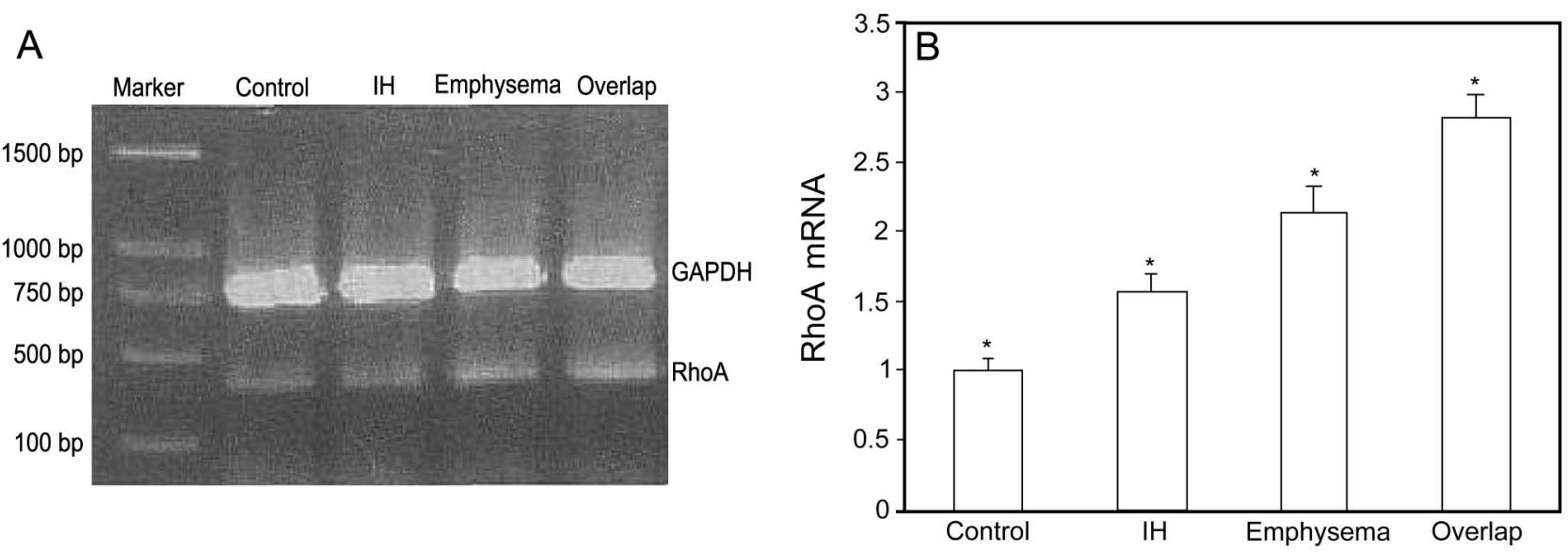

Fig. 4. RhoA mRNA expression levels of endothelial cells from right common carotid artery performed with reverse transcription polymerase chain reaction. ${ }^{*} P<.05$ compared with other group. $\mathrm{IH}=$ intermittent hypoxia; bp, base pair; RhoA = Ras homology A; GAPDH, glyceraldehyde-3-phosphate dehydrogenase.

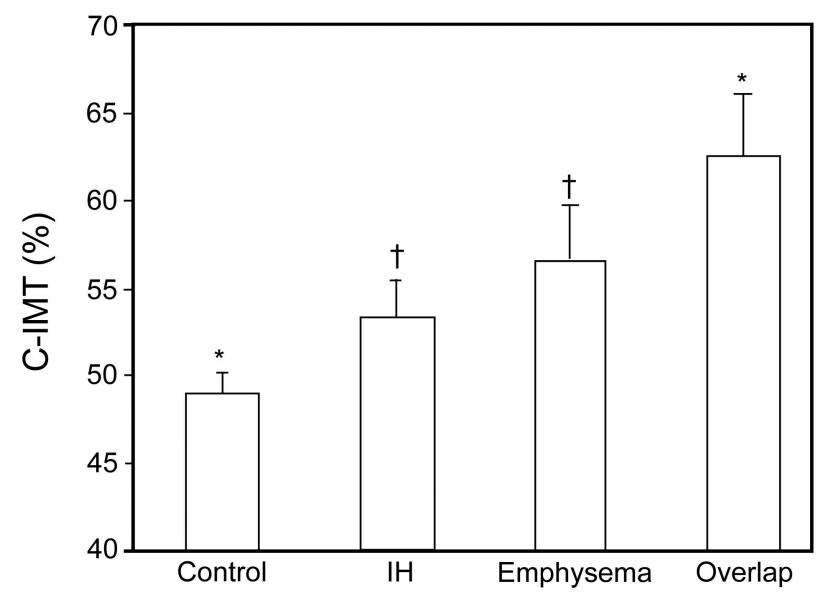

Fig. 5. C-IMT (\%) of right carotid artery from animals. ${ }^{*} P<.05$ compared with other group. † No statistical difference between control and IH groups. C-IMT $(\%)=$ ratio of carotid intima-media thickness to whole thickness of the vessel; $\mathrm{IH}=$ intermittent hypoxia.

lowest in the control group $(0.13 \pm 0.06$, all $P<.001)$, and those percentages were higher in the $\mathrm{IH}(0.57 \pm 0.07)$ group when compared with the emphysema group $(0.23 \pm 0.06, P<.001)$. See Figure 6, B and D.

\section{Discussion}

Both COPD and OSA are independent risk factors for atherosclerosis and cardiovascular diseases, and their coexistence in OS probably increases this risk. The underlying mechanisms are still unclear, but may involve systematic inflammation, endothelial dysfunction, and tonic elevation of sympathetic neural activity. ${ }^{20}$ Inflammation plays a key role in atherosclerotic plaque formation, from initiation of the fatty streak to the later stages of plaque rupture..$^{10}$

Systematic inflammation in OS may launch nuclear factor- $\kappa \mathrm{B}$-dependent pathways involving TNF- $\alpha$ and IL-6, providing insight into potential interactions between both disorders. TNF- $\alpha$ and IL- 6 are main pro-inflammatory cytokines associated with atherosclerosis and consequent cardiovascular diseases. ${ }^{21}$ Circulating IL-6 and TNF- $\alpha$ levels correlate well with early atherosclerosis, and are predictive of coronary heart disease and congestive heart failure. ${ }^{22}$ Various pro-inflammatory cytokines have been reported to induce monocyte migration into the endothelium and subendothelial spaces, and induce the release of related factors that promote smooth muscle cell migration and proliferation, which may synthesize and deposit extracellular matrix. ${ }^{22}$ Through this process, monocytes become foam cells and contribute to the progression of atherosclerosis. Therefore, the endothelial dysfunction associated with OS is most probably the result of initiation and propagation of oxidative stress and inflammatory responses within the microvasculature. ${ }^{23}$ Studies found increased IL-6 and TNF- $\alpha$ levels in OSA. ${ }^{10}$ Additionally, in COPD, IL-6 and TNF- $\alpha$ levels are also increased, particularly during exacerbations. ${ }^{20}$ From our study, overlap exposure may elicit a more severe systematic inflammatory response, which is consistent with one of our previous reports assessing systematic inflammation and oxidative stress in the liver of a model of sleep-related hypoxemia in emphysematous rats. ${ }^{14}$

Systematic hypoxemia contributes to TNF- $\alpha$ elevations in COPD and OSA, and these elevations are independent from obesity. ${ }^{24}$ It may be particularly relevant in OS, where hypoxemia is more pronounced. Furthermore, circulating TNF- $\alpha$ levels are higher in COPD patients with pulmonary hypertension, who are more hypoxemic. ${ }^{25}$ Thus, TNF- $\alpha$ and related pro-inflammatory cytokine levels should be 
A
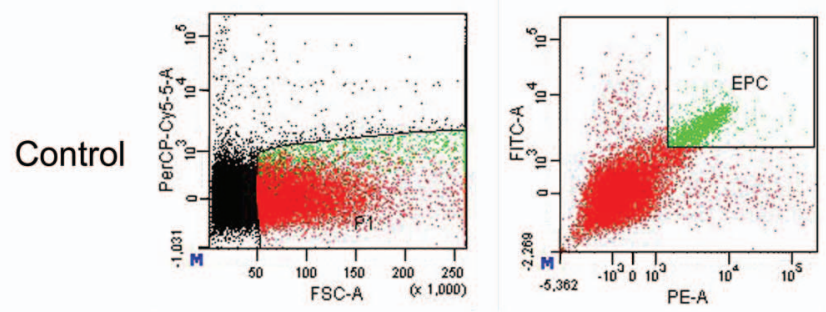

$\mathrm{IH}$
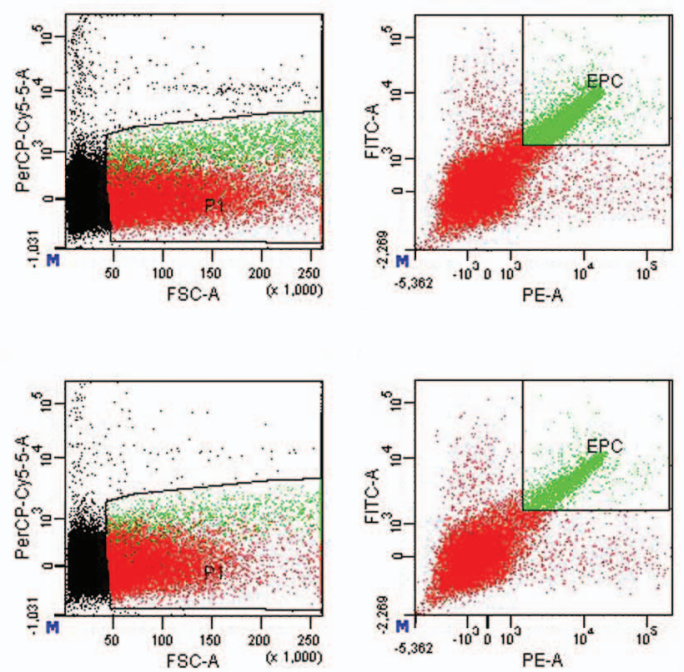

Emphysema
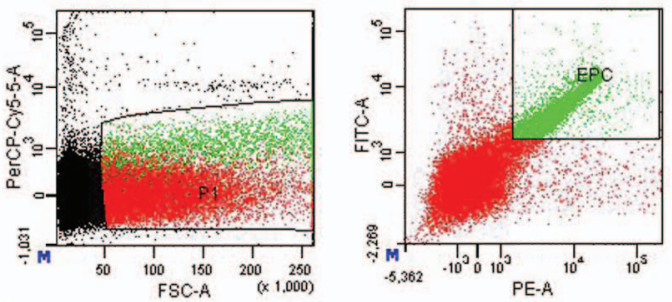

B
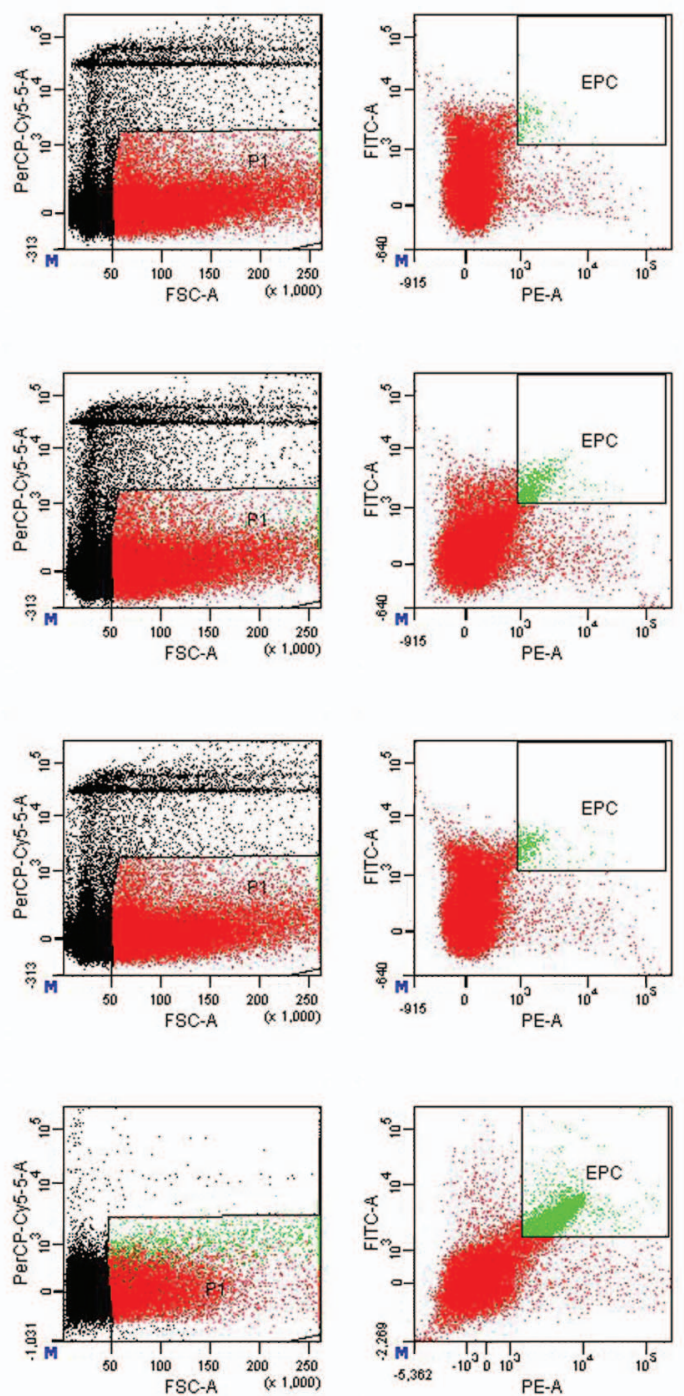

C

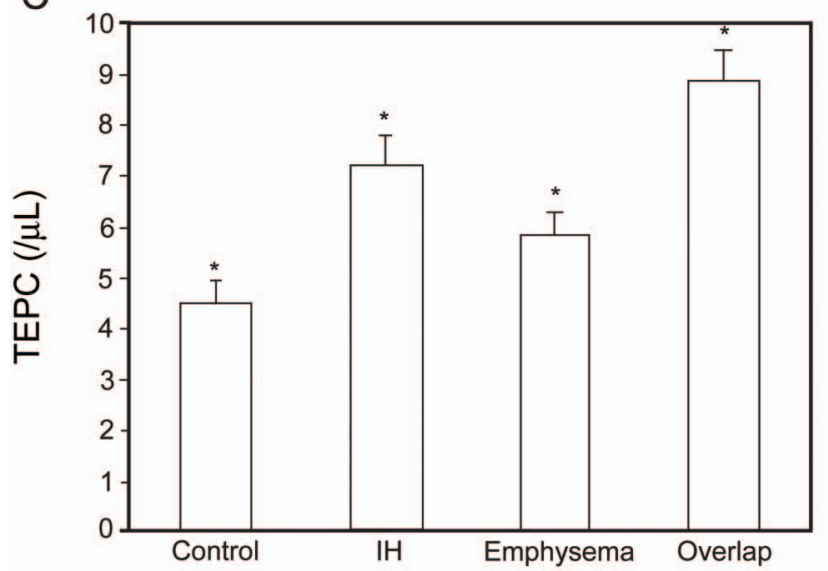

D

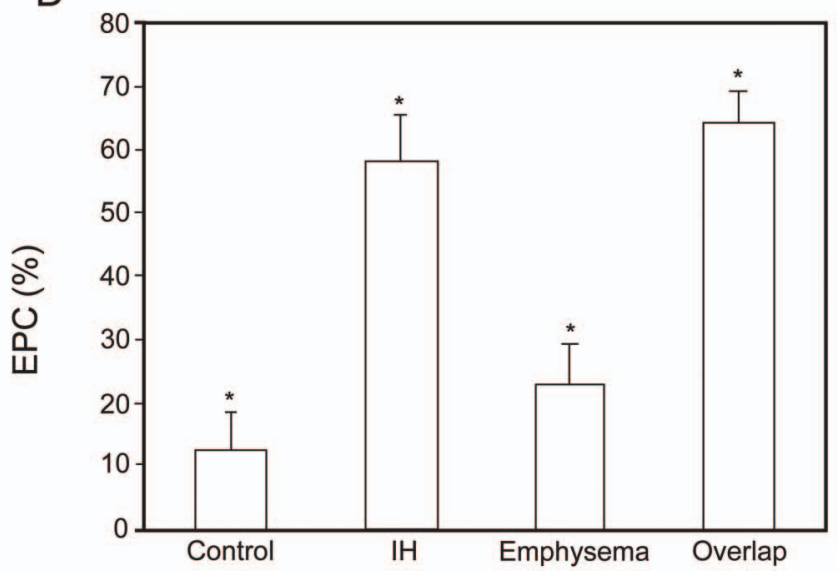

Fig. 6. Flow cytometric analysis of surface markers of EPCs from rat peripheral blood $(A$ and $C)$ and rat bone marrow $(B$ and $D)$. ${ }^{\star} P<.05$ compared with other group. PerCP = peridinin chlorophyll protein; Cy $5=$ cyanidin 5 ; FITC = fluorescein isothiocyanate; $P E=$ phycoerythrin; FSC = forward scatter; EPC = endothelial progenitor cell; IH = intermittent hypoxia. 
particularly elevated in patients with the OS, ${ }^{10}$ where nocturnal tonic desaturations associated with IH may have overlapped effects on increased TNF- $\alpha$ levels. ${ }^{24}$

From our results, IH and smoking exposure, overlapped directly or synergistically, cause a more severe RhoA activation in the overlap group, which may result in a more obvious transendothelial migration of monocytes as its clinical implication. The hallmark for the development of atherosclerosis is the adhesion of monocytes to endothelium, followed by transendothelial migration of monocytes. ${ }^{23}$ There is growing evidence that the RhoA/Rho-associated kinase (ROCK) pathway has an important pathophysiological role in vascular endothelial dysfunction. ${ }^{26}$ Activated RhoA shrinks endothelial cells and increases cell permeability, making it more feasible for inflammatory cells, like leukocytes, to pass through the endothelial barrier when it combines with elevated adhesive capability caused by cellular inflammation. ${ }^{6}$ One of our previous studies suggests that IH may cause endothelial damage through activated IL-6 and RhoA in OSA patients. ${ }^{6}$ Moreover, in another study, it has been demonstrated that the RhoA, with its downstream effector Rho kinase, has been involved in systematic endothelial dysfunction in smokers with normal lung function. The authors found GTP-RhoA protein expression was significantly increased in patients with COPD. ${ }^{27}$ Hypoxia might play a potential role in the upregulation of RhoA/ROCK pathway in COPD, and the increase in RhoA/ROCK activity associated with chronic hypoxia has already been demonstrated in animal models of pulmonary hypertension. ${ }^{28}$ Although the mechanisms through which the RhoA/ROCK pathway is activated in chronic hypoxia have not been well understood, reactive oxygen species are involved in the activation of multiple intracellular signaling cascades including the RhoA/ROCK pathway, where the precise mechanisms require further investigation. ${ }^{27}$

Subclinical carotid atherosclerosis is reported to correlate well with coronary and intracranial atherosclerosis and is therefore a predictor of future cardiovascular events. An important tool for evaluating early impairment of the vascular structures leading toward a systemic atherosclerotic process is the IMT evaluation. ${ }^{29}$ In our study, C-IMT values are increased in the IH and the emphysema group, whereas those in the overlap group are the highest, which suggests clinically that patients with the combination of COPD and OSA may have a worse vascular prognosis than patients who have only one of these diseases. Within the vasculature, the release of pro-inflammatory cytokines, chemokines, and growth factors by inflammatory cells causes proliferation and migration of smooth muscle cells from the media to the intima, as well as recruitment of new immunoinflammatory cells from circulation. Mariani et al ${ }^{30}$ found a significant correlation between C-IMT and OSA severity, and patients with more severe OSA have higher
C-IMT compared with mild and moderate OSA. In OSA, IH, hypercapnia, micro-arousals, sympathetic hyperactivity, oxidative stress, systematic inflammation, and hypercoagulability are all potential mechanisms that may play roles in the development of increased C-IMT and endothelial dysfunction, although only IH was simulated in our model rats. ${ }^{30}$ One of our previous studies suggests that $\mathrm{IH}$ may cause the increase of IMT, ${ }^{6}$ and there is substantial evidence that IH in rodents plays a specific role in promoting cellular changes at the vascular wall, thus triggering atherosclerosis. ${ }^{30,31}$ Furthermore, Ozgen Alpaydin et $\mathrm{al}^{32}$ observed increased C-IMT in patients with COPD compared with controls after adjusting for smoking, hypertension, fasting plasma glucose, triglyceride, and high density lipoprotein cholesterol levels. This finding might suggest that patients with air flow obstruction are more prone to atherogenesis due to some other mechanisms apart from smoking. ${ }^{32}$ This emphasizes the importance of timely diagnosis and treatment of coexistent COPD and OSA for preventing the development of severe cardiovascular complications.

The discovery of EPC has revolutionized the horizons of regenerative cardiovascular medicine. EPC is believed to reflect another aspect of endothelial function and refers to the group of circulating cells that mediate endothelial repair at sites of vascular injury directly by differentiation into mature endothelial cells, or indirectly by secreting vasoactive substances promoting repair or mediating angiogenesis at sites of ischemia. It is believed that, under homeostatic conditions, these cells are released from the bone marrow, circulate in the blood, and contribute to the repairing of endothelium in response to general wear and tear. ${ }^{33}$ For some diseases, such as vascular injury in atherosclerosis and ischemia, EPCs isolated from autologous bone marrow could be induced to differentiate in vitro and could be transplanted into the diseased lesion, which would eliminate the risk of rejection. ${ }^{19}$ EPC levels, both in bone marrow or in peripheral blood circulation, are highest in the overlap group, indicating a robust mobilization when these rats are exposed to overlap treatment. However, this robust mobilization does not mean a robust repairing capability. The actual population of circulating EPCs represents between $0.0001 \%$ and $0.05 \%$ of total white blood cells in the peripheral blood. Such diversity can result from differences in antibody affinity or the health status of an individual. ${ }^{34}$ In contrast, under inflammatory conditions, their enforced mobilization and activation may tip the balance from stimulating tissue repair to promoting tissue remodeling and fibrosis. After being released from bone marrow, they undergo further differentiation with a sequence of changes in their surface antigen (cluster of differentiation, $\mathrm{CD}$ ), the pattern of which forms the basis of identifying circulating EPC by fluorescence-activated cell sorting analysis. CD133+ and kinase insert domain 
receptor $(\mathrm{KDR})+$ or vascular endothelial growth factor receptor (VEGFR)2+ cells represent the pool of early pluripotent stem cells, which have angiogenic potential and show good correlation with cardiovascular risk estimates. ${ }^{33}$

Several previous studies have investigated the association between OSA and EPC. Findings have been controversial, with negative, ${ }^{35}$ neutral, ${ }^{36}$ and positive results. ${ }^{33} \mathrm{~A}$ possible reason for this discrepancy may be that these studies were performed on only a small number of participants and/or different markers were used to characterize these cells. EPCs may be mobilized endogenously in response to tissue ischemia and hypoxia, as well as by mediators and signaling pathways such as vascular endothelial growth factor (VEGF) and hypoxia-inducible factor- $1 \alpha$, which could be activated by IH. IH may contribute to cardioprotection and the development of collateral vessels by mobilizing progenitor cells to the circulation and damaged myocardium. ${ }^{34}$ Additionally, several studies demonstrate that both pulmonary and systematic vascular alterations in patients with COPD closely relate to endothelial dysfunction and inflammation. ${ }^{37,38}$ In patients with COPD, levels of circulating CD34+ cells are reduced compared with normal control subjects, and it has been suggested, although not proven, that this may reflect the increased trafficking of progenitor cells into the lungs. Levels of circulating CD34+ cells appear to increase during exacerbations of COPD, which has been correlated with an elevation in the plasma levels of VEGF from these patients. As it is known that VEGF can stimulate the mobilization of EPCs from the bone marrow into the circulation, these 2 observations may be functionally related. ${ }^{39}$

According to ABG results obtained from preliminary experiments and pathologic characteristics of smoke exposure rats, we have made a rat model of IH in emphysematous rats (caused by exposure to cigarette smoke for a long period of time and identified with pathologic criteria). ${ }^{8}$ One advantage of the animal model used in this study is that, by noninvasively applying recurrent $\mathrm{IH}$ in healthy animals, the rats were subjected to periodic stimuli very similar (in magnitude, duration, and frequency) to the ones experienced by patients with OSA. As a partial model of sleep apnea, IH allows testing of the specific role of IH on endothelial dysfunction and assessment of both plasma and tissue alterations that may contribute to atherosclerosis development and progression. This acute model allowed us to study the early effects of these injurious stimuli while avoiding other confounding factors found in OSA patients, which also cause inflammation and endothelial dysfunction (such as metabolic syndrome, obesity, hypertension). Our OS model, in fact, is the combination of emphysema and IH, not a real overlap syndrome, and cannot mimic the OS patients completely, which is a shortcoming of our experiment. This limitation does not, however, affect the aim of this study. It should also be pointed out that investigators in different studies assessed circulating EPCs through different methods. Whereas some studies assessed circulating EPCs using flow cytometry alone, ${ }^{35,40}$ another study assessed circulating EPCs by the assay of endothelial colony-forming units. ${ }^{35}$ In addition, the EPCs studied by one group are not necessarily the same cell type as those of another. One of the major limitations in studying EPCs is the lack of unifying phenotypic markers that are employed by different investigators. Thus, different investigators employ different marker combinations for the assessment of EPCs: CD34+ VEGFR2+, $\mathrm{CD} 34+133+$, or CD34+ CD133 + VEGFR2 +. ${ }^{15}$ A more feasible and readily applicable method to investigate potential adaptation mechanisms in response to a long-term challenge as in OS patients and to study the role of EPCs in vascular repair remains to be studied.

\section{Conclusions}

Regardless of whether the 2 diseases are mechanistically linked, OS patients have more severe nocturnal hypoxemia and pulmonary hypertension than patients with OSA or COPD alone, which probably has important clinical consequences. It was reported that OS patients have greater mortality compared with patients who have either OSA or COPD alone. ${ }^{10}$ These clinical findings may be the implications of our bench study. Understanding EPC biology in OS would contribute to the knowledge of vascular pathogenesis, as well as potential therapeutic interventions to treat cardiovascular complications in patients with OS.

\section{ACKNOWLEDGMENTS}

We are grateful for information and correction from Jau-Shyong Hong $\mathrm{PhD}$ (Neuropharmacology Section, Laboratory of Toxicology and Pharmacology, National Institute of Environmental Health Sciences, National Institutes of Health, Research Triangle Park, North Carolina) and from Danielle Speer RPN and Ambrose Chiang MD (Division of Pulmonary and Critical Care Medicine, Duke University Medical Center, Durham, North Carolina).

\section{REFERENCES}

1. Marin JM, Soriano JB, Carrizo SJ, Boldova A, Celli BR. Outcomes in patients with chronic obstructive pulmonary disease and obstructive sleep apnea: the overlap syndrome. Am J Respir Crit Care Med 2010;182(3):325-331.

2. Greenberg-Dotan S, Reuveni H, Tal A, Oksenberg A, Cohen A, Shaya FT, et al. Increased prevalence of obstructive lung disease in patients with obstructive sleep apnea. Sleep Breath 2014;18(1):6975.

3. Ganga HV, Nair SU, Puppala VK, Miller WL. Risk of new-onset atrial fibrillation in elderly patients with the overlap syndrome: a retrospective cohort study. J Geriatr Cardiol 2013;10(2):129-134.

4. Owens RL, Malhotra A. Sleep-disordered breathing and COPD: the overlap syndrome. Respir Care 2010;55(10):1333-1344; discussion 1344-1346. 


\section{INFLAMMATION AND EPCS IN EMPHYSEMATOUS RATS}

5. Feng J, Chen BY, Cui LY. Carotid body-mediated changes of sympathetic nerve and their relationships with hypertension. Chin Med J (Engl) 2008;121(17):1732-1735.

6. Feng J, Chen BY, Cui LY, Wang BL, Liu CX, Chen PF, et al. Inflammation status of rabbit carotid artery model endothelium during intermittent hypoxia exposure and its relationship with leptin. Sleep Breath 2009;13(3):277-283.

7. Feng J, Chiang AA, Wu Q, Chen BY, Cui LY, Liang DC, et al. Sleep-related hypoxemia aggravates systematic inflammation in emphysematous rats. Chin Med J (Engl) 2010;123(17):2392-2399.

8. Feng J, Wang QS, Chiang A, Chen BY. The effects of sleep hypoxia on coagulant factors and hepatic inflammation in emphysematous rats. PLoS One 2010;5(10):e13201. doi: 10.1371/ journal.pone.0013201.

9. Yang Q, Wang Y, Feng J, Cao J, Chen B. Intermittent hypoxia from obstructive sleep apnea may cause neuronal impairment and dysfunction in central nervous system: the potential roles played by microglia. Neuropsychiatr Dis Treat 2013;9:1077-1086.

10. McNicholas WT. Chronic obstructive pulmonary disease and obstructive sleep apnea: overlaps in pathophysiology, systemic inflammation, and cardiovascular disease. Am J Respir Crit Care Med 2009;180(8):692-700.

11. Bishop AL, Hall A. Rho GTPases and their effector proteins. Biochem J 2000;348 Pt 2:241-255.

12. Berger S, Aronson D, Lavie P, Lavie L. Endothelial progenitor cells in acute myocardial infarction and sleep-disordered breathing. Am J Respir Crit Care Med 2013;187(1):90-98.

13. Finkelstein J, Cha E, Scharf SM. Chronic obstructive pulmonary disease as an independent risk factor for cardiovascular morbidity. Int J Chron Obstruct Pulmon Dis 2009;4:337-349.

14. Ioachimescu OC, Teodorescu M. Integrating the overlap of obstructive lung disease and obstructive sleep apnoea: OLDOSA syndrome. Respirology 2013;18(3):421-431.

15. Wang Q, Wu Q, Feng J, Sun X. Obstructive sleep apnea and endothelial progenitor cells. Patient Prefer Adherence 2013;7:1077-1090.

16. Kilkenny C, Browne W, Cuthill IC, Emerson M, Altman DG, NC3Rs Reporting Guidelines Working Group. Animal research: reporting in vivo experiments: the ARRIVE guidelines. J Gene Med 2010;12(7): 561-563.

17. NC3Rs Reporting Guidelines Working Group. Animal research: reporting in vivo experiments: the ARRIVE guidelines. Exp Physiol 2010;95(8):842-844.

18. Horner RL, Sanford LD, Annis D, Pack AI, Morrison AR. Serotonin at the laterodorsal tegmental nucleus suppresses rapid-eye-movement sleep in freely behaving rats. J Neurosci 1997;17(19):75417552.

19. Yang N, Li D, Jiao P, Chen B, Yao S, Sang H, et al. The characteristics of endothelial progenitor cells derived from mononuclear cells of rat bone marrow in different culture conditions. Cytotechnology 2011;63(3):217-226.

20. Zamarrón C, García Paz V, Morete E, del Campo Matías F. Association of chronic obstructive pulmonary disease and obstructive sleep apnea consequences. Int J Chron Obstruct Pulmon Dis 2008; 3(4):671-682.

21. Li Y, Schwabe RF, DeVries-Seimon T, Yao PM, Gerbod-Giannone MC, Tall AR, et al. Free cholesterol-loaded macrophages are an abundant source of tumor necrosis factor-alpha and interleukin-6: model of NF-kappaB- and map kinase-dependent inflammation in advanced atherosclerosis. J Biol Chem 2005;280(23):21763-21772.

22. Tamaki S, Yamauchi M, Fukuoka A, Makinodan K, Koyama N, Tomoda $\mathrm{K}$, et al. Production of inflammatory mediators by monocytes in patients with obstructive sleep apnea syndrome. Intern Med 2009;48(15):1255-1262.
23. Rajesh M, Mukhopadhyay P, Bátkai S, Haskó G, Liaudet L, Huffman JW, et al. CB2-receptor stimulation attenuates TNF-alpha-induced human endothelial cell activation, transendothelial migration of monocytes, and monocyte-endothelial adhesion. Am J Physiol Heart Circ Physiol 2007;293(4):H2210-H2218.

24. Lacedonia D, Salerno FG, Sabato R, Carpagnano GE, Aliani M, Palladino GP, Barbaro MP. Airway cell patterns in patients suffering from COPD and OSAS (overlap syndrome). Respir Med 2011;105(2): 303-309.

25. Joppa P, Petrasova D, Stancak B, Tkacova R. Systemic inflammation in patients with COPD and pulmonary hypertension. Chest 2006; 130(2):326-333.

26. Yao L, Romero MJ, Toque HA, Yang G, Caldwell RB, Caldwell RW. The role of RhoA/Rho kinase pathway in endothelial dysfunction. J Cardiovasc Dis Res 2010;1(4):165-170.

27. Bei Y, Duong-Quy S, Hua-Huy T, Dao P, Le-Dong NN, Dinh-Xuan AT. Activation of RhoA/Rho-kinase pathway accounts for pulmonary endothelial dysfunction in patients with chronic obstructive pulmonary disease. Physiol Rep 2013;1(5):e00105. doi: 10.1002/ phy 2.105

28. Hyvelin JM, Howell K, Nichol A, Costello CM, Preston RJ, McLoughlin P. Inhibition of Rho-kinase attenuates hypoxia-induced angiogenesis in the pulmonary circulation. Circ Res 2005;97(2):185-191.

29. Ciccone MM, Scicchitano P, Mitacchione G, Zito A, Gesualdo M, Caputo $\mathrm{P}$, et al. Is there a correlation between OSAS duration/severity and carotid intima-media thickness? Respir Med 2012;106(5): 740-746.

30. Mariani S, Fiore D, Barbaro G, Basciani S, Saponara M, D'Arcangelo E, et al. Association of epicardial fat thickness with the severity of obstructive sleep apnea in obese patients. Int J Cardiol 2013;167(5): 2244-2249.

31. Lévy P, Pépin JL, Arnaud C, Baguet JP, Dematteis M, Mach F. Obstructive sleep apnea and atherosclerosis. Prog Cardiovasc Dis 2009;51(5):400-410

32. Ozgen Alpaydin A, Konyar Arslan I, Serter S, Sakar Coskun A, Celik P, Taneli F, et al. Metabolic syndrome and carotid intimamedia thickness in chronic obstructive pulmonary disease. Multidiscip Respir Med 2013;8(1):61

33. Lui MM, Tse HF, Mak JC, Lam JC, Lam DC, Tan KC, Ip MS. Altered profile of circulating endothelial progenitor cells in obstructive sleep apnea. Sleep Breath 2013;17(3):937-942.

34. Berger S, Lavie L. Endothelial progenitor cells in cardiovascular disease and hypoxia-potential implications to obstructive sleep apnea. Transl Res 2011;158(1):1-13.

35. Yun CH, Jung KH, Chu K, Kim SH, Ji KH, Park HK, et al. Increased circulating endothelial microparticles and carotid atherosclerosis in obstructive sleep apnea. J Clin Neurol 2010;6(2):89-98.

36. Kizawa T, Nakamura Y, Takahashi S, Sakurai S, Yamauchi K, Inoue H. Pathogenic role of angiotensin II and oxidised LDL in obstructive sleep apnoea. Eur Respir J 2009;34(6):1390-1398.

37. Alpsoy S, Akyuz A, Mutlu LC, Oran M, Akkoyun DC, Topcu B, et al. Serum fetuin-A levels are associated with carotid intima media thickness in patients with normotensive chronic obstructive pulmonary disease. Cardiol J 2014;21(2):191-197.

38. Matsuoka S, Yamashiro T, Diaz A, Estépar RS, Ross JC, Silverman EK, et al. The relationship between small pulmonary vascular alteration and aortic atherosclerosis in chronic obstructive pulmonary disease: quantitative CT analysis. Acad Radiol 2011;18(1):40-46.

39. Jones CP, Rankin SM. Bone marrow-derived stem cells and respiratory disease. Chest 2011;140(1):205-211.

40. Murri M, García-Delgado R, Alcázar-Ramírez J, Fernández-Ramos A, Alcaide J, Cardona F, Tinahones FJ. Effect of CPAP on oxidative stress and circulating progenitor cell levels in sleep patients with apnea-hypopnea syndrome. Respir Care 2011;56(11):1830-1836. 